\title{
A Chebyshev Polynomial Method for Optimal Control with State Constraints*
}

\author{
JACQUES VLASSENBROECK $\dagger$
}

\begin{abstract}
A numerical algorithm based on a Chebyshev series expansion of control and state variables solves optimal control problems with state inequality constraints by replacing the original continuous-time problem by a finitedimensional non-linear programming problem.
\end{abstract}

Key Words-Optimal control; Chebyshev approximation; parameter optimization; inequality constraints.

\begin{abstract}
This paper presents a numerical technique for solving non-linear constrained optimal control problems. The method extends previous contributions to non-linear unconstrained optimal control problems and is based upon a Chebyshev series expansion of state and control. The differential and integral expressions from the system dynamics and the performance index, the boundary conditions and other general conditions are converted into some algebraic equations. State inequality constraints are transformed into equality constraints through the use of slack variables. The technique may start from a feasible or non-feasible trajectory and avoids problems of singular arcs. The applicability is illustrated on two well-known state variable inequality constrained optimal control problems. Extensions of the approach to problems with other equality and inequality constraints on state and control are described but have not yet been tested on practical examples.
\end{abstract}

\section{INTRODUCTION}

DURING the past 25 years many practical computing techniques have been developed in optimal control. Most of these methods successfully solve the unconstrained problem, but the presence of, for example, state variable inequality constraints (SVIC) often resulted in both analytical and computational difficulties. Theoretical aspects of the SVIC problem have been studied by Berkovitz (1961), Dreyfus (1962), Chang (1962), Berkovitz and Dreyfus (1965) and Speyer and Bryson (1968), while early contributions to the numerical computation were due to Dreyfus (1962), Kelley (1962), Bryson and Denham (1964), McGill (1965), Lasdon et al. (1967), Jacobson and Lele (1969), Mehra and Davis (1972), Neuman and Sen (1973) and

\footnotetext{
* Received 25 September 1986; revised 4 November 1987; revised 3 December 1987; received in final form 14 December 1987. The original version of this paper was not presented at any IFAC meeting. This paper was recommended for publication in revised form by Associate Editor E. Kreindler under the direction of Editor H. Kwakernaak.

$\dagger$ Department of Analytical Mechanics, Vrije Universiteit Brussel, Pleinlaan 2, 1050 Brussels, Belgium.
}

Maurer and Gillessen (1975). The approaches of Dreyfus (1962) and Bryson and Denham (1964) require guesses for both the number and location of the junction points between constrained and unconstrained arcs. Integral penalty functions still provide good practical means for solving SVIC problems. Such problems may turn out to be the last redoubt of the penalty-function technique (Kelley, 1962; McGill, 1965; Lasdon et al., 1967). For refinement of penaltyapproximation solutions one turns to the indirect variational method and multiple-shooting (Maurer and Gillessen, 1975). Jacobson and Lele (1969) used a slack variable to transform a SVIC problem into an unconstrained problem of higher dimension for which the optimal trajectory exhibits singular arcs. Mehra and Davis (1972) described that difficulties arising from handling SVIC are due to the exclusive use of control variables as independent variables and presented the so-called generalized gradient technique. Finally, Neuman and Sen (1973) introduced an algorithm for solving linearquadratic SVIC problems through collocation and approximation of state and control by cubic splines.

Earlier, a Chebyshev algorithm for unconstrained problems was presented and successfully applied on several non-linear problems with non-linear boundary conditions, and fixed and variable final time (Van Dooren and Vlassenbroeck, 1980, 1981, 1982; Vlassenbroeck et al., 1981). This technique is briefly described in Appendix $A$ and will be extended here in order to solve non-linear optimal control problems with SVIC.

Vlach (1969) stated that Chebyshev polynomials of the first class can uniformly approximate a much broader class of functions than other 
ultraspherical polynomials. Therefore, and because of their favourable numerical properties (Vlach, 1969; Fox and Parker, 1972; Dahlquist and Bjorck, 1974), the current approach is based upon the expansion of both the state and control in Chebyshev series having unknown coefficients. However, the idea of using both the state and control as independent variables is not new (Canon et al., 1970; Tabak and Kuo, 1971), and polynomial expansion of control and/or state has already been used (Ghonaimy and Bernholtz, 1966; Johnson, 1969; Fegley et al., 1971; Neuman and Sen, 1973; Sirisena, 1973; Nair, 1978).

The technique converts differential and integral expressions from the system dynamics and the performance index, the boundary conditions and other general constraints into systems of (non-linear) algebraic equations in the unknown coefficients (and system parameters, eventually). The SVIC are transformed into equality constraints through the use of slack variables (Berkovitz, 1961; Jacobson and Lele, 1969) and can be replaced by a set of algebraic equations. The technique may start from a feasible or non-feasible trajectory and has the advantage of avoiding problems of singular arcs which arise for portions of the state trajectory that lie along the constraint boundary.

The algorithm is illustrated on a linearquadratic optimal control problem, treated initially without and afterwards with first and second-order SVIC. For the unconstrained problem the resulting equations are linear and it will be seen that the Chebyshev technique gives better results than the modified steepest descent (Hsieh, 1965) or collocation and approximation by cubic splines (Neuman and Sen, 1973). The results for the two SVIC problems are comparable to or better than those reported by Jacobson and Lele (1969), Mehra and Davis (1972) and Neuman and Sen (1973).

\section{PROBLEM STATEMENT}

The aim is to minimize the performance index

$$
I=H(X(T))+\int_{0}^{\mathrm{T}} G(X, U, \tau) \mathrm{d} \tau
$$

subject to the system dynamics represented by the following non-linear ordinary differential equation

$$
\frac{\mathrm{d} X}{\mathrm{~d} \tau}=F(X, U, \tau), \quad 0 \leqslant \tau \leqslant T \text { (fixed), }
$$

the initial condition

$$
X(0)=X_{0}
$$

and the SVIC

$$
S(X, \tau) \leqslant 0, \quad 0 \leqslant \tau \leqslant T .
$$

In general, $X$ and $F$ are $s$-dimensional, $U$ is $r$-dimensional, $S$ is $q$-dimensional and $H$ and $G$ are scalar functions. $F, G, H$ and $S$ may be non-linear.

If SVIC (4) is of $p$ th order, which means that the $p$ th time derivative of $S$ is the first to contain the control $U$ explicitly, then Jacobson and Lele (1969) have $2 p$ additional state-costate differential equations. They assume that $r \geqslant q$ and that $X(0)$ satisfies (4) with strict inequality because numerical problems can be expected if $X(0)$ is (very) close to this boundary since $S(X(0), 0)$ appears in the denumerator of some generated initial conditions. Neither of these assumptions have to be considered here.

For simplicity the case with $s=r=q=1$ is used, but generalization is readily straightforward. Cases where the final time $T$ is free and/or where there exist other general (non-linear) boundary conditions such as

$$
\Psi(X(0), X(T))+\int_{0}^{T} \phi(X, U, \tau) \mathrm{d} \tau=0
$$

require only minor modifications (see Appendix A) and are also discussed and illustrated in earlier work (Vlassenbroeck et al., 1981; Van Dooren and Vlassenbroeck, 1981).

Other constraints of the form

$$
\begin{array}{cc}
C(U, \tau)=0, & C(X, U, \tau)=0, \quad S(X, \tau)=0, \\
C(U, \tau) \leqslant 0, & C(X, U, \tau) \leqslant 0, \\
0 \leqslant \tau \leqslant T,
\end{array}
$$

are considered in Appendix B.

\section{CHEBYSHEV APPROXIMATIONS}

In order to use Chebyshev polynomials of the first class, $\left\{T_{n}(t)=\cos n\left(\cos ^{-1} t\right)\right\}$, which are defined on $t \in(-1,1), \tau \in(0, T)$ is transformed into $t \in(-1,1)$ by using the transformation

$$
\tau=\frac{T}{2}(1+t)
$$

which transforms (1)-(4) into

$$
\begin{gathered}
I=h(x(1))+\int_{-1}^{1} g(x, u, t) \mathrm{d} t \\
\frac{\mathrm{d} x}{\mathrm{~d} t}=f(x, u, t), \quad-1 \leqslant t \leqslant 1, \\
x(-1)=x_{-1}=X_{0}, \\
s(x(t), t) \leqslant 0, \quad-1 \leqslant t \leqslant 1 .
\end{gathered}
$$

The approximate solution of this optimal control problem is represented by a Chebyshev 
series of order $m$ for both the state and control and is given by

$$
\begin{aligned}
& x_{m}(t)=\frac{1}{2} a_{0} T_{0}(t)+\sum_{n=1}^{m} a_{n} T_{n}(t), \\
& u_{m}(t)=\frac{1}{2} b_{0} T_{0}(t)+\sum_{n=1}^{m} b_{n} T_{n}(t),
\end{aligned}
$$

where $\alpha \equiv\left(a_{0}, \ldots, a_{m}\right), \beta \equiv\left(b_{0}, \ldots, b_{m}\right)$ are unknown. The technique from Appendix $A$ gives then

$$
\begin{aligned}
J(\alpha, \beta)=\frac{1}{2} C_{0}(\alpha) & +\sum_{n=1}^{m_{1}} C_{n}(\alpha)+B_{0}(\alpha, \beta) \\
& -\sum_{n=1}^{m_{1}} \frac{1+(-1)^{n}}{n^{2}-1} B_{n}(\alpha, \beta)
\end{aligned}
$$

for the approximation of the performance index (9),

$$
\begin{aligned}
& E_{0}(\alpha, \beta) \equiv A_{0}(\alpha, \beta)-A_{2}(\alpha, \beta)-2 a_{1}=0, \\
& E_{1}(\alpha, \beta) \equiv A_{1}(\alpha, \beta)-A_{3}(\alpha, \beta)-4 a_{2}=0, \\
& \vdots \\
& E_{M}(\alpha, \beta) \equiv A_{M}(\alpha, \beta)=0,
\end{aligned}
$$

for the approximation of the system dynamics (10) and

$$
E_{M+1}(\alpha) \equiv \frac{1}{2} a_{0}+\sum_{n=1}^{m}(-1)^{n} a_{n}-x_{-1}=0
$$

for the initial condition (11).

A slack variable $y(t)$ is used to convert the inequality constraint (12) into the following equality constraint

$$
s(x(t), t)=-\frac{1}{2} y^{2}(t), \quad-1 \leqslant t \leqslant 1 .
$$

$y(t)$ is approximated by a Chebyshev series of order $m$ with unknown coefficients $\left\{r_{n}\right\}$, i.e.

$$
y_{m}(t)=\frac{1}{2} r_{0} T_{0}(t)+\sum_{n=1}^{m} r_{n} T_{n}(t),
$$

where $\rho \equiv\left(r_{0}, \ldots, r_{m}\right)$ are unknown. Substituting (13) and (19) into (18) and applying the Chebyshev balance principle, which consists of equating equal-order terms, yields

$$
D_{n}(\alpha, \rho)=0, \quad\left(n=0,1, \ldots, m_{2}\right),
$$

where $\left\{D_{n}\right\}$ are the coefficients of the Chebyshev series expansion of $s\left(x_{m}(t), t\right)+\frac{1}{2} y_{m}^{2}(t)$, truncated after the term of order $m_{2}$. They are calculated very accurately by means of the following formula (Fox and Parker, 1972):

$$
\begin{aligned}
D_{n}(\alpha, \rho)=\frac{2}{K} \sum_{i=1}^{K}\left[s\left(x_{m}\left(\cos \theta_{i}\right), \cos \theta_{i}\right)\right. & \\
\left.+\frac{1}{2} y_{m}^{2}\left(\cos \theta_{i}\right)\right] \cos n \theta_{i}, & \left(n=0,1, \ldots, m_{2}\right), \\
K>m_{2}, \quad \theta_{i} & =\frac{2 i-1}{K} \cdot \frac{\pi}{2},
\end{aligned}
$$

The optimal control problem with SVIC can now be replaced by the following parameter optimization problem: find $\alpha, \beta$ and $\rho$ such that $J(\alpha, \beta)$ from $(15)$ is minimal subject to the constraints (16), (17) and (20). Now any of many mathematical programming techniques can be applied to solve this constrained minimization problem. The solution proposed by Lagrange is to form an unconstrained problem by appending the constraints to the performance index by means of the Lagrange multipliers. If the following is defined

$$
\begin{array}{r}
L(\alpha, \beta, \rho, \lambda)=J(\alpha, \beta)+\sum_{v=0}^{M+1} \lambda_{v} E_{v}(\alpha, \beta) \\
+\sum_{v=0}^{m_{2}} \lambda_{v+M+2} D_{v}(\alpha, \rho)
\end{array}
$$

where $\lambda$ represent the unknown Lagrange multipliers, then the necessary conditions for stationarity are given by

$$
\begin{gathered}
\frac{\partial L}{\partial a_{\mu}}=0, \quad \frac{\partial L}{\partial b_{\mu}}=0, \quad \frac{\partial L}{\partial r_{\mu}}=0, \\
(\mu=0,1, \ldots, m), \\
E_{v}=0, \quad(v=0,1, \ldots, M+1), \\
D_{v}=0, \quad\left(v=0,1, \ldots, m_{2}\right) .
\end{gathered}
$$

The iterative Newton method or a modified version for better convergence (Dahlquist and Bjorck, 1974) can now be applied to solve (23)-(25).

Remark. The use of slack variables could be avoided by using the following technique. Substituting $x_{m}(t)$ from (13) into (12) and calculating this new expression in a number of points $t_{i},-1=t_{0}<t_{1}<\cdots<t_{K^{\prime}}=1$, yields

$$
D_{v}(\alpha) \leqslant 0, \quad\left(v=0,1, \ldots, K^{\prime}\right),
$$

and the new problem would then be as follows: find $\alpha$ and $\beta$ that minimize $J$ such that $\left\{E_{v}=0\right\}$ and $\left\{D_{v} \leqslant 0\right\}$ are satisfied. This technique was applied by Neuman and Sen (1973) on linear-quadratic optimal control problems with linear SVIC. Another possibility to avoid slack variables is offered through the penalty function technique described by Kelley (1962) and McGill (1965) that involves the use of the Heaviside step function.

\section{THEORETICAL AND COMPUTATIONAL CONSIDERATIONS}

For simplicity the same degree of expansion was used for the state, control and slack variable 
in (13), (14) and (19) respectively though it would be more general not to do so. The choice of $m$ depends upon the required accuracy and the error of approximation on $x(t)$ and $u(t)$ equals $\sum_{n=m+1}^{\infty} a_{n} T_{n}(t)$ and $\sum_{n=m+1}^{\infty} b_{n} T_{n}(t)$ respectively. If the number of terms is increased, the approximation will improve and will tend to the exact solution. Due to the use of orthogonal polynomials for representing the state and the control, increasing $m$ does not result in an appreciable change in the low-order coefficients.

The choice of $m_{1}$ and $m_{2}$ should logically depend upon the form of $h, g$ and $s$. However, this choice is not critical at all and generally, $m_{1}=2 m$ and $m_{2}=m$, are used; and to ensure that a feasible trajectory is obtained, for moderate approximation for state and control, the given SVIC (12) is modified into

$$
s(x(t), t)+\delta \leqslant 0, \quad-1 \leqslant t \leqslant 1,
$$

where the constant $\delta$ is modified throughout the iterative process such that the state trajectory (nearly) touches the boundary from within the feasible region.

However, there is no rule for determining the parameter values $m$ and $K$ in order to reach a given accuracy, and a theoretical error estimation for the performance index and the state and control variables is not given. This disadvantage is, however, shared by almost every other existing method for solving problems with SVIC.

\section{EXAMPLES}

The method illustrated above was applied to a linear-quadratic optimal control problem, treated initially without and afterwards with firstand second-order SVIC and for which results from alternative techniques are presented in the literature.

\section{Example 1}

Find $U(\tau)$ that minimizes

$$
I=\int_{0}^{1}\left(X_{1}^{2}+X_{2}^{2}+0.005 U^{2}\right) \mathrm{d} \tau
$$

subject to

$$
\begin{aligned}
& \dot{X}_{1}=X_{2}, \quad 0 \leqslant \tau \leqslant 1 \\
& \dot{X}_{2}=-X_{2}+U,
\end{aligned}
$$

and

$$
\begin{aligned}
& X_{1}(0)=0, \\
& X_{2}(0)=-1 .
\end{aligned}
$$

This example, for which the exact solution can be found by Pontryagin's maximum principle method, has been attempted by Hsieh (1965) using a modified steepest descent method and by
Neuman and Sen (1973) using collocation and approximation by cubic splines. The determining equations in the Chebyshev technique are linear in the unknowns so that they are immediately solved and it was shown that the exact solution for the state and control variables, as well as for the performance index, can be approximated within any given precision. The results from Hsieh (1965) and Neuman and Sen (1973) for the performance index are compared to those obtained by the Chebyshev technique and to the exact value in Table 1. It can be seen that the Chebyshev technique with $m=9$ already offers a very precise solution (deviation from the exact value is approximately $0.01 \%$ ) which is much better than the results of Hsieh (1965) and Neuman and Sen (1973) for which the case $N=9$ has much more unknowns (cubic splines in each of the $N+1$ intervals).

\section{Example 2}

Find $U(\tau)$ that minimizes (28) subject to (29)-(32) and the following first-order SVIC

$$
X_{2}(\tau)-8(\tau-0.5)^{2}+0.5 \leqslant 0, \quad 0 \leqslant \tau \leqslant 1 .
$$

\section{Example 3}

Find $U(\tau)$ that minimizes (28) subject to (29)-(32) and the following second-order SVIC

$$
X_{1}(\tau)-8(\tau-0.5)^{2}+0.5 \leqslant 0, \quad 0 \leqslant \tau \leqslant 1 .
$$

Examples 2 and 3 were solved by Jacobson and Lele (1969), Mehra and Davis (1972) and Neuman and Sen (1973).

For the Chebyshev technique the next two sets of starting values were chosen very arbitrarily: (i) $\bar{x}_{1}(t)=-0.25, \bar{x}_{2}(t)=-0.25, \bar{u}(t)=0.5$ and $\bar{y}(t)=0.5$; (ii) $\bar{x}_{1}(t)=0.5, \bar{x}_{2}(t)=0.5, \bar{u}(t)=1.0$ and $\dot{y}(t)=1.0$, for $-1 \leqslant t \leqslant 1$. In all the experiments $m_{1}$ was set equal to $2 m$ and $m_{2}$ to $m$. The procedure from Appendix A to obtain starting values for the Lagrange multipliers was not followed but $\lambda_{i}$ was chosen to be 0.1 , for all

TABle 1. Minimum VAlues of the PERFormanCE INDEX For EXAMPLE 1

\begin{tabular}{llc}
\hline \multicolumn{1}{c}{ Source } & \multicolumn{1}{c}{$J$} & $\begin{array}{c}\text { Deviation from } \\
\text { exact value }\end{array}$ \\
\hline Exact value & 0.06936094 & \\
Hsieh (1965) & 0.0702 & $8.4 \times 10^{-4}$ \\
Neuman and Sen (1973) & & \\
$\quad N=4$ & 0.0703 & $9.4 \times 10^{-4}$ \\
$\quad N=9$ & 0.06989 & $5.3 \times 10^{-4}$ \\
Chebyshev technique & & \\
$m=5$ & 0.07595 & $6.6 \times 10^{-3}$ \\
$m=9$ & 0.069368 & $7.1 \times 10^{-6}$ \\
$m=10$ & 0.06936186 & $9.2 \times 10^{-7}$ \\
$m=11$ & 0.06936103 & $9.0 \times 10^{-8}$ \\
$m=12$ & 0.06936095 & $1.0 \times 10^{-8}$ \\
$m=13$ & 0.06936094 & $<10^{-8}$ \\
\hline
\end{tabular}


TABLE 2. MiNimUM VALUES OF THE PERFORMANCE INDEX for EXAMPLES 2 AND 3

\begin{tabular}{lcc}
\hline \multicolumn{1}{c}{ Source } & $\begin{array}{c}\text { Example 2 } \\
\text { (first-order SVIC) }\end{array}$ & $\begin{array}{c}\text { Example 3 } \\
\text { (second-order SVIC) }\end{array}$ \\
\hline $\begin{array}{l}\text { Jacobson and Lele (1969) } \\
\text { Mehra and Davis (1972) }\end{array}$ & 0.164 & 0.75 \\
Neuman and Sen (1973) & 0.178 & 0.79 \\
$\quad \begin{array}{l}N=4 \\
N=9\end{array}$ & 0.1642 & 0.5422 \\
Chebyshev technique & 0.16946 & 0.6894 \\
$m=5, K=12$ & & \\
$m=9, K=20$ & 0.196 & 0.766 \\
$m=10, K=22$ & 0.1870 & 0.7483 \\
$m=11, K=24$ & 0.1788 & 0.7456 \\
$m=12, K=26$ & 0.17784 & 0.74522 \\
$m=13, K=28$ & 0.17358 & 0.74101 \\
& 0.17185 & 0.74096 \\
\hline
\end{tabular}

$i$, thereby facilitating programming work but increasing somewhat the total number of iterations. For $m=5$ and $K=12$, starting from (i), the final results were obtained after $4.6 \mathrm{~s}$ computer time on a CDC Cyber $170 / 750$ for Example 2 and $6.6 \mathrm{~s}$ for Example 3. Starting from (ii), 5.3 and $7.3 \mathrm{~s}$ respectively were needed. It must be emphasized that these two sets of starting values do not satisfy the given differential equations, initial conditions and the considered SVIC. Also other sets did lead to exactly the same results: $J=0.196$ for Example 2 and 0.766 for Example 3. For Example 2 with $m=5$ and starting from (i), the algorithm ran successively for $\delta=0$ (eight iterations), $\delta=$ 0.030 (three iterations) and $\delta=0.028$ (two iterations), and thus only two shifts* on the SVIC were needed to ensure that the trajectory nowhere violated the given SVIC and that it touched the constraint from within the feasible region. From these results it also emerged that for $\delta=0$ the maximum violation of the constraint, i.e. the largest positive value of $s(\cdot)$ over the entire interval, and the performance index amounted to 0.03 and 0.180 respectively. For $\delta=0.030$ the SVIC was satisfied [even $s(\cdot)<0]$ but $J$ had increased to 0.197 and, finally, for $\delta=0.028$ the trajectory for $x_{2}$ (nearly) touches the boundary constraint for $t=-0.273$ (or $\tau=0.364$ ) and $J$ equals 0.196 . So moving away from the constraint boundary but into the feasible region makes $J$ increase and therefore the lowest performance index should be sought with those methods that allow the state trajectory to lie on or touch the boundary constraint from within the feasible region. It is essentially for this reason that the technique was refined by adjoining the parameter $\delta$ such that for all $m$ a feasible trajectory with lowest performance index can be obtained. If, for

\footnotetext{
* Determined through the calculation of the SVIC in 50 points, uniformly distributed over the interval.
}

$m=5$, the parameter $K$ is increased from 12 to 16 , it has no effect on the first six decimals of the results while the computer time increases from 4.6 to $4.8 \mathrm{~s}$ (Example 2).

For higher-order approximations the previous results can now be used as starting values. The 13th-order approximation is calculated within 21.4 and $31.0 \mathrm{~s}$ for Examples 2 and 3 respectively. For Example 2, it was found that the distance between $X_{2}$ and the boundary was less than $10^{-2}$ for $0.28<\tau<0.68$ and that $X_{2}$ touched the boundary at $\tau=0.333[S(0.333)=$ $-0.0001]$. For Example 3, the distance between $X_{1}$ and the boundary was less than $10^{-2}$ for $0.44<\tau<0.52$ and $X_{1}$ touched the boundary at $\tau=0.481[S(0.481)=-0.0004]$. Because of the higher order of the approximation only one (small) shift on $\delta$ was sufficient: $\delta=0.0035$ for Example 2 and 0.012 for Example 3.

The results from Jacobson and Lele (1969), Mehra and Davis (1972) and Neuman and Sen (1973) for the performance index can be compared to the present results in Table $2, \dagger$ from which it can be seen that Neuman and Sen (1973) offer the lowest performance index. However, their SVIC are severely violated (Neuman and Sen, 1973), on the contrary, the other methods generate solutions that satisfy the imposed SVIC. To make a fair comparison, not only should the violation or satisfaction of the SVIC be examined but the errors on the differential equations and initial conditions should also be computed.

From Table 2, it can be concluded that the present technique gives better or comparable results, and, as for Example 1, the errors in the differential equations (29) and (30) and in the initial conditions (31) and (32) are negligible.

The results for the control and state variables for the three examples are graphed in Figs 1-3

† The content of Table 2 is due to Neuman and Sen (1973) and was completed with our results. 


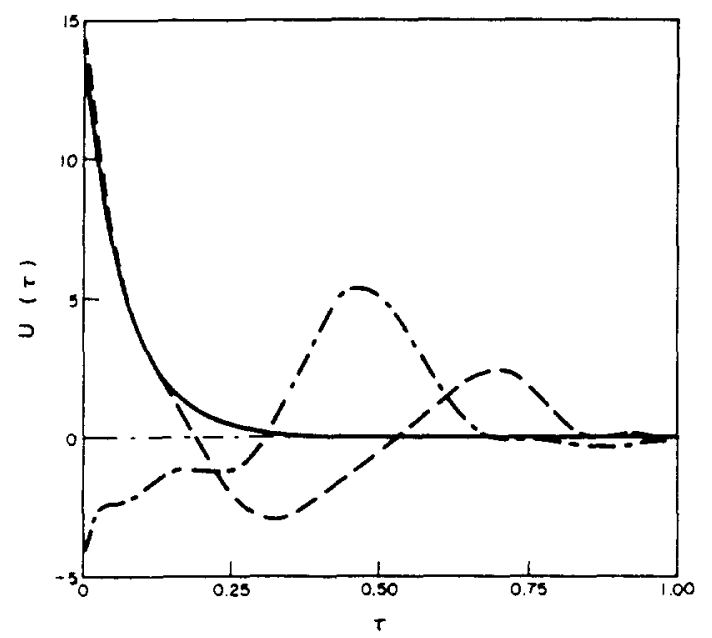

FIG. 1. The control variable for Example $1(-)$, Example $2(----)$ and Example $3(-\cdot-\cdot)$.

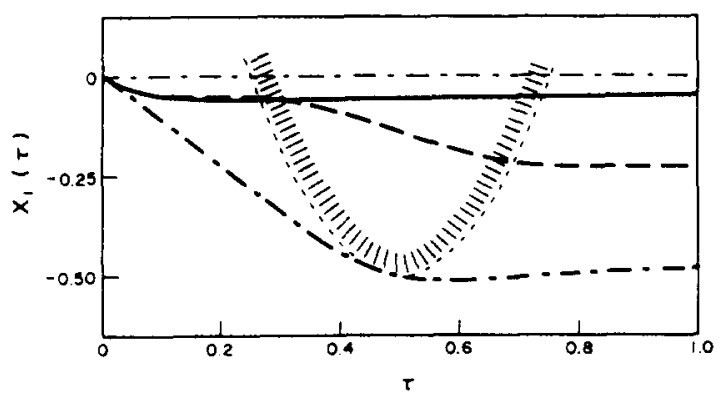

FIG. 2. The state variable $X_{1}$ for Example 1 ( $\longrightarrow$ ), Example $2(---)$ and Example $3(-\cdot--)$.

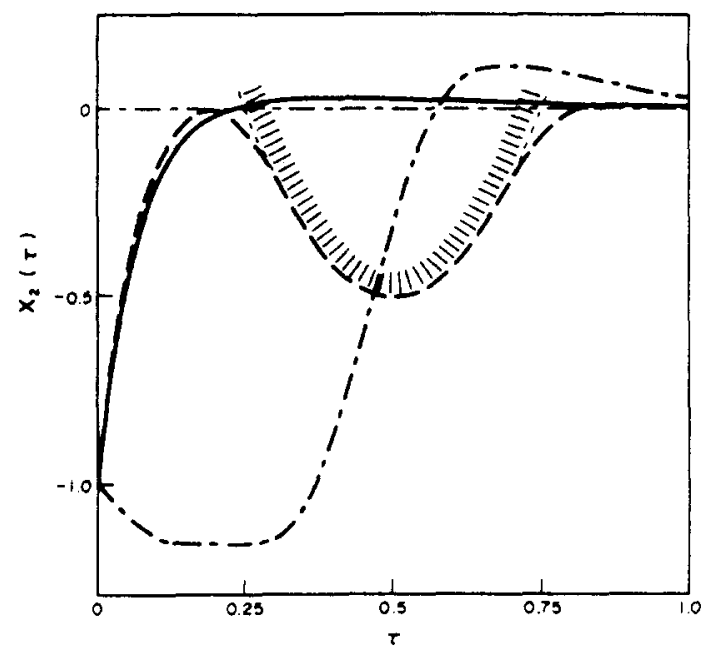

Fig. 3. The state variable $X_{2}$ for Example 1 ( $(-)$, Example $2(----)$ and Example $3(-\cdot--)$.

for the case $m=13$. In Fig. 4 are graphed the slack variable for Examples 2 and 3, though in some cases, depending upon the initial values that were used, the reflected image with respect to the time axis was obtained. This is quite normal since it is the square of the slack variable that is used in (18).

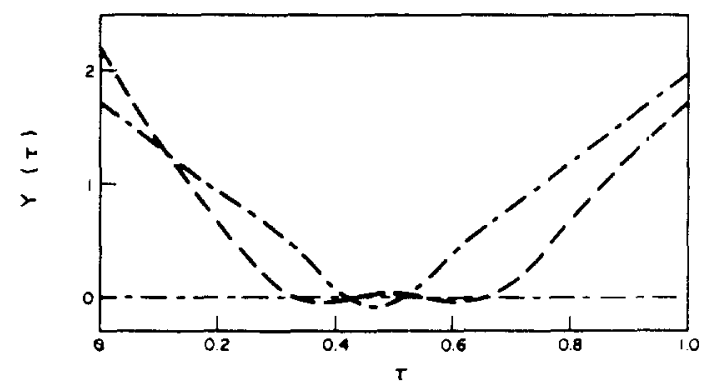

FIG. 4. The slack variable for Example $2(---)$ and Example $3(-\cdot-\cdot)$.

\section{CONCLUSIONS}

A method for solving non-linear optimal control problems with SVIC has been outlined. The technique is based upon the use of a Chebyshev series expansion for the state and control variables, and the SVIC is removed through the use of a slack variable. The proposed method needs no previous elimination of the control variable, starts from a feasible or non-feasible trajectory and avoids problems of singular arcs. It can generate a trajectory for which the SVIC is nowhere violated.

The applicability is illustrated on a linearquadratic optimal control problem with first- and second-order SVIC. The results obtained were better than or comparable to those obtained by some existing techniques.

Also described in this paper is how other equality and inequality constraints on state and/or control should be handled, while the extension to multidimensional optimal control problems with several SVIC is straightforward.

\section{REFERENCES}

Berkovitz, L. D. (1961). Variational methods in problems of control and programming. J. Math. Anal. Applic., 3, 145. Berkovitz, L. D. and S. E. Dreyfus (1965). The equivalence of some necessary conditions for optimal control in problems with bounded state variables. J. Math. Anal. Applic., 10, 275.

Bryson, A. E. and W. Denham (1964). Optimal programming with inequality constraints. II. Solution by steepest ascent. AIAA Jl, 2, 25.

Canon, M. D., C. D. Cullum, Jr and E. Polak (1970). Theory of Optimal Control and Mathematical Programming. McGraw-Hill, New York.

Chang, S. S. L. (1962). Optimal control in bounded state space. Automatica, 1, 55 .

Dahlquist, G. and A. Bjorck (1974). Numerical Methods. Prentice-Hall, Englewood Cliffs, NJ.

Dreyfus, S. E. (1962). Variational problems with state variable inequality constraints. J. Math. Anal. Applic., 4, 297.

Fegley, K. A., S. Blum, J. O. Bergholm, A. J. Calise, J. E. Marowitz, G. Porcelli and L. P. Sinha (1971). Stochastic and deterministic design and control via linear and quadratic programming. IEEE Trans. Aut. Control, AC.16, 759.

Fox, L. and I. B. Parker (1972). Chebysheu Polynomials in Numerical Analysis. Oxford University Press, Oxford.

Ghonaimy, M. A. R. and B. Bernholtz (1966). On a direct 
method of optimization: linear control systems. Proc. IIIrd IFAC, Vol. 1.

Hsieh, H. C. (1965). Synthesis of adaptive control systems by function space methods. In $\mathrm{C}$. $\mathrm{T}$. Leondes (Ed.), Advances in Control Systems, Vol. 2, pp. 117-208. Academic Press, New York.

Jacobson, D. H. and M. M. Lele (1969). A transformation technique for optimal control problems with a state variable inequality constraint. IEEE Trans. Aut. Control, AC-14, 457 .

Johnson, F. T. (1969). Approximate finite-thrust trajectory optimization. AIAA Jl, 7, 993.

Kelley, H. J. (1962). Method of gradients. In G. Leitmann (Ed.), Optimization Techniques, pp. 205-254. Academic Press, New York.

Lasdon, L. S., A. D. Waren and R. K. Rice (1967). An interior penalty method for inequality constrained optimal control problems. IEEE Trans. Aut. Control, AC-12, 388.

Maurer, H. and W. Gillessen (1975). Application of multiple shooting to the numerical solution of optimal control problems with bounded state variables. Computing, 15, 105.

McGill, R. (1965). Optimal control, inequality state constraints and the generalized Newton-Raphson algorithm. SIAM J. Control, 3, 291.

Mehra, R. K. and R. E. Davis (1972). A generalized gradient method for optimal control problems with inequality constraints and singular arcs. IEEE Trans. Aut. Control, AC-17, 69.

Nair, G. G. (1978). Suboptimal control of nonlinear systems. Automatica, 14, 517.

Neuman, C. P. and A. Sen (1973). A suboptimal control algorithm for constrained problems using cubic splines. Automatica, 9, 601

Sirisena, H. R. (1973). Computation of optimal controls using a piecewise polynomial parameterization. IEEE Trans. Aut. Control, AC-18, 409.

Speyer, J. L. and A. E. Bryson (1968). Solution of optimal programming problems with state variable inequality constraints by direct adjoining. Preprints JACC. Ann Arbor, Michigan, p. 485.

Tabak, D. and B. C. Kuo (1971). Optimal Control by Mathematical Programming. Prentice-Hall, Englewood Cliffs, NJ.

Urabe, M. (1967). Numerical solution of multi-point boundary value problems in Chebyshev series. Theory of the method. Numer. Math., 9, 341.

Urabe, M. (1969). Numerical solution of boundary value problems in Chebyshev series. A method of computation and error estimation. Lecture Notes in Mathematics, Vol. 109 , p. 40. Springer, Berlin.

Van Dooren, R. and J. Vlassenbroeck (1980). A new look at the brachistochrone problem. Z. Angew. Math. Phys., 31, 785.

Van Dooren, R. and J. Vlassenbroeck (1981). A computational method in optimal systems control with various applications. In Marshall, J. E., W. D. Collins, C. J. Harris and D. H. Owens (Eds), Third IMA Conference on Control Theory, pp. 407-429. Academic Press, London.

Van Dooren, R. and J. Vlassenbroeck (1982). Chebyshev series solution of the controlled Duffing oscillator. $J$. Comput. Phys., 47, 321.

Vlach, J. (1969). Computerized Approximation and Synthesis of Linear Networks. Wiley, London.

Vlassenbroeck, J., H. Janssen and R. Van Dooren (1981). A direct Chebyshev approach with practical applicability in optimal control problems. Proc. VIIIth Triennial World Congress IFAC, Vol. 3, pp. 159-164.

Walsh, G. R. (1975). Methods of Optimization. Wiley, London.

\section{APPENDIX A}

The Chebyshev technique for unconstrained optimal control problems

The purpose of this Appendix is to describe briefly the Chebyshev technique for the following non-linear uncon- strained optimal control problem: find the control $u(t)$ such that the performance index (9) is minimized subject to the system dynamics (10) and the initial condition (11).

Substituting the Chebyshev approximations for the state and control from (13) and (14) into (9), setting $\left\{B_{n}(\alpha, \beta)\right\}$ and $\left\{C_{n}(\alpha)\right\}$ to be the Chebyshev coefficients of $g\left[x_{m}(t), u_{m}(t), t\right]$ and $h\left[x_{m}(t)\right]$ respectively, and using the property that (Fox and Parker, 1972)

and

$$
T_{n}(1)=1, \quad(n=0,1, \ldots)
$$

if

$$
\begin{gathered}
\int_{-1}^{1} q(t) \mathrm{d} t=q_{0}-\sum_{n=2}^{\infty} \frac{1+(-1)^{n}}{n^{2}-1} q_{n} \\
q(t)=\frac{1}{2} q_{0} T_{0}(t)+\sum_{n=1}^{\infty} q_{n} T_{n}(t)
\end{gathered}
$$

the following approximation for $l$ is obtained

$$
\begin{aligned}
J(\alpha, \beta)=\frac{1}{2} C_{0}(\alpha)+\sum_{n=1}^{\infty} C_{n}(\alpha) & +B_{0}(\alpha, \beta) \\
& -\sum_{n=2}^{\infty} \frac{1+(-1)^{n}}{n^{2}-1} B_{n}(\alpha, \beta) .
\end{aligned}
$$

For computational reasons the infinite series is truncated at order $m_{1}$ and we calculate $\left\{B_{n}\right\}$ and $\left\{C_{n}\right\}$ by means of the following formula (Fox and Parker, 1972):

$$
\begin{aligned}
B_{n}(\alpha, \beta) & =\frac{2}{K} \sum_{i=1}^{K} g\left[x_{m}\left(\cos \theta_{i}\right), u_{m}\left(\cos \theta_{i}\right), \cos \theta_{i}\right] \cos n \theta_{i}, \\
C_{n}(\alpha) & =\frac{2}{K} \sum_{i=1}^{K} h\left[x_{m}\left(\cos \theta_{i}\right)\right] \cos n \theta_{i}, \\
(n & \left.=0,1, \ldots, m_{1}\right), \quad K>m_{1}, \quad \theta_{i}=\frac{2 i-1}{K} \cdot \frac{\pi}{2}
\end{aligned}
$$

where $t_{i}=\cos \theta_{i}$ are the roots of the Chebyshev polynomial of order $K$.

If (13) and (14) are substituted into (10) and if the Chebyshev series expansion of the right-hand side of $(10)$ is truncated after the term of order $M$, the following is obtained:

$$
\frac{\mathrm{d} x_{m}}{\mathrm{~d} t}=f_{M}\left(x_{m}(t), u_{m}(t), t\right)
$$

where

$$
f_{M}\left(x_{m}(t), u_{m}(t), t\right)=\frac{1}{2} A_{0}(\alpha, \beta) T_{0}(t)+\sum_{n=1}^{M} A_{n}(\alpha, \beta) T_{n}(t)
$$

with

$$
\begin{gathered}
A_{n}(\alpha, \beta)=\frac{2}{K} \sum_{i=1}^{K} f\left(x_{m}\left(\cos \theta_{i}\right), u_{m}\left(\cos \theta_{i}\right), \cos \theta_{i}\right) \cos n \theta_{i} \\
(n=0,1, \ldots, M), \quad K>M, \quad \theta_{i}=\frac{2 i-1}{K} \cdot \frac{\pi}{2} .
\end{gathered}
$$

The left-hand side of (A5) is of degree $m-1$ while on the right-hand side the polynomial is of degree $M$. If $f$ is non-linear $M$ is set equal to $m-1$ after Urabe $(1967,1969)$, but if $f$ is linear $M$ is set equal to $m$. If $\left\{a_{n}^{\prime}\right\}$ represent the Chebyshev coefficients of $\mathrm{dx} x_{m} / \mathrm{d} t$ then equating the coefficients of same-order Chebyshev polynomials yields:

$$
\begin{aligned}
a_{0}^{\prime} & =A_{0}, \quad a_{1}^{\prime}=A_{1}, \ldots, a_{m-1}^{\prime}=A_{m-1}, \\
0 & =A_{m}, \ldots, 0=A_{M} .
\end{aligned}
$$

The relationship between $\left\{a_{n}^{\prime}\right\}$ and $\left\{a_{n}\right\}$ can be expressed by (Fox and Parker, 1972)

$$
a_{n-1}^{\prime}-a_{n+1}^{\prime}-2 n a_{n}=0, \quad(n=1,2, \ldots),
$$

which, with (A7), gives the following relationships in $\alpha$ and $\beta$ :

$$
\begin{array}{r}
E_{n-1}(\alpha, \beta) \equiv A_{n-1}(\alpha, \beta)-A_{n+1}(\alpha, \beta)-2 n a_{n}=0, \\
(n=1,2, \ldots, m), \\
E_{n-1}(\alpha, \beta) \equiv A_{n-1}(\alpha, \beta)-A_{n+1}(\alpha, \beta)=0, \\
(n=m+1, \ldots, M+1),
\end{array}
$$


with

$$
A_{M+1}(\alpha, \beta)=A_{M+2}(\alpha, \beta)=0 .
$$

These equations are the approximations for the system dynamics.

If (13) is substituted in (11) and the property that (Fox and Parker, 1972)

$$
T_{n}(-1)=(-1)^{n}, \quad(n=0,1, \ldots),
$$

is used, the following is obtained

$$
E_{M+1}(\alpha) \equiv \frac{1}{2} a_{0}+\sum_{n=1}^{m}(-1)^{n} a_{n}-x_{-1}=0
$$

as an approximation for the initial condition (11).

The original problem to minimize (9) subject to (10) and (11) can now be replaced by the following parameter optimization problem: find $\alpha$ and $\beta$ such that $J(\alpha, \beta)$ from (A3) is minimized subject to the constraints (A9) and (A11). The method of Lagrange (Walsh, 1975) defines

$$
L(\alpha, \beta, \lambda)=J(\alpha, \beta)+\sum_{v=0}^{M+1} \lambda_{v} E_{v}(\alpha, \beta)
$$

where $\lambda \equiv\left(\lambda_{0}, \ldots, \lambda_{M+1}\right)$ represent the Lagrange multipliers and the necessary conditions for stationarity are given by

$$
\begin{array}{rlrl}
\frac{\partial L}{\partial a_{\mu}}=0, & & \frac{\partial L}{\partial b_{\mu}}=0, & (\mu=0,1, \ldots, m), \\
E_{v}=0, & (v=0,1, \ldots, M+1) .
\end{array}
$$

Sufficient conditions for a local minimum are the stationarity conditions (A13) and (A14) and the convexity condition expressing the positive definiteness of a certain quadratic form. Equations (A13) and (A14) can be solved by means of the iterative Newton method. Starting values for $\left\{a_{n}\right\}$ and $\left\{b_{n}\right\}$ are usually obtained from some physical insight in the problem or by applying the proposed method for very low order. Starting values for $\left\{\lambda_{n}\right\}$ can then be obtained by selecting arbitrarily $M+2$ equations from (A13), and solving this linear system in $\left\{\lambda_{n}\right\}$.

If the final time, $T$, is free, then an additional equation is given by

$$
\frac{\partial L}{\partial T}=0
$$

Constraints of the form (5) or even general multipoint boundary conditions which could severely burden many numerical methods, are treated like the expression (1)

Remark. The constrained parameter optimization problem could be converted into an unconstrained problem by using a penalty function technique, thus avoiding enhancing the dimensionality of the problem.

\section{APPENDIX B}

Equality and inequality constraints on state and control Equality constraints of the form

$c(u(t), t)=0, \quad c(x(t), u(t), t)=0 \quad$ or $\quad s(x(t), t)=0$,

can be treated like the system dynamics: we substitute $x_{m}(t)$ and/or $u_{m}(t)$ from (13) and (14) in the given constraint from (B1), expand $c(\cdot)$ and/or $s(\cdot)$ in Chebyshev series and apply the Chebyshev balance principle. The resulting constraints in $\alpha$ and/or $\beta$ are then adjoined to the other constraints.

For inequality constraints of the form

$$
c(u(t), t) \leqslant 0 \text { or } c(x(t), u(t), t) \leqslant 0
$$

a treatment similar to that applied to SVIC is suggested. First convert the inequality constraint from (B2) into the following equality constraint using a slack variable:

$$
c(\cdot)=-\frac{1}{2} y^{2}(t) \text {. }
$$

Equations (19), (13) and/or (14) are then substituted into (B3) and the Chebyshev balance principle is applied.

Up to now only SVIC problems have been numerically examined, but problems with constraints of the form (B1) and/or (B2) are expected to give equally good results.

Remark. For some problems where the resulting solution is of the bang-bang type, it is recommended that the original interval $\tau \in(0, T)$ be divided into several subintervals depending upon the number of discontinuities, and the instants at which the discontinuities occur be treated as additional unknowns. Every subinterval is transformed into $t \in(-1,1)$ on which the proposed technique is then applied. Expressing the continuity of the state variables at the unknown instants gives additional constraint relations. This technique was applied to the problem of the double integrator with the constraint $-1 \leqslant u \leqslant 1$ for which the bang-bang solution for $u(t)$ and the solution for $x(t)$ were successfully calculated. 